\title{
Monitoring circulating epithelial tumour cells (CETC) to gauge therapy: in patients with disease progression after trastuzumab persisting CETC can be eliminated by combined lapatinib treatment
}

\author{
Oumar Camara • Cornelia Jörke · Ulrike Hammer • \\ Anne Egbe • Carola Rabenstein · Ingo B. Runnebaum • \\ Klaus Hoeffken • Katharina Pachmann \\ Received: 3 April 2008 / Accepted: 24 September 2008 / Published online: 21 October 2008 \\ (C) The Author(s) 2008. This article is published with open access at Springerlink.com
}

\begin{abstract}
Background In breast cancers, the gene for the growth factor receptor HER2 can be amplified leading to increased aggressiveness and metastasis formation. The monoclonal antibody trastuzumab prolongs relapse-free survival highly significantly but eventually many patients relapse.

Method In this study, CETC were monitored using the Maintrac ${ }^{\circledR}$ method during adjuvant trastuzumab treatment and during subsequent treatment with capecitabine/lapatinib.

Results In one patient, trastuzumab led to marginal reduction in CETC with disease progress. The combination of capecitabine/lapatinib was preliminarily capable to eliminate all CETC, however, CETC reappeared. The second patient received adjuvant taxane together with trastuzumab and 1 year of further trastuzumab during which CETC increased. After stopping trastuzumab skin metastases occurred. Capecitabine/lapatinib led to complete CETC elimination with stable disease.

Conclusions In patients with lack of CETC reduction in spite of trastuzumab treatment correlated with disease progression the combination of capecitabine/lapatinib highly efficiently led to rapid elimination of CETC warranting further monitoring during such studies.
\end{abstract}

O. Camara · A. Egbe $\cdot$ I. B. Runnebaum

Women's Hospital, Friedrich Schiller University,

Bachstr. 18, 07740 Jena, Germany

C. Jörke · U. Hammer · K. Hoeffken · K. Pachmann $(\square)$

Department of Experimental Hematology and Oncology,

Clinic for Internal Medicine II, Friedrich Schiller Universität Jena,

Erlanger Allee 101, 07747 Jena, Germany

e-mail: katharina.pachmann@med.uni-jena.de

C. Rabenstein $\cdot$ K. Pachmann

Transfusionsmedizinisches Zentrum Bayreuth,

Kurpromenade 2, 95448 Bayreuth, Germany
Keywords Circulating epithelial tumour cells . Breast cancer therapy $\cdot$ Trastuzumab $\cdot$ Lapatinib

\section{Purpose}

The HER2/neu gene, coding for a growth factor receptor which is over-expressed in these patients, is amplified in approximately $25 \%$ of breast cancer patients, showing highly aggressive disease. Trastuzumab is a monoclonal antibody targeted to the external domain of this growth factor receptor and has been shown to be effective in metastatic breast cancer (Seidman et al. 2001), with clinical benefit seen in about half of the patients with HER2/neupositive disease (Piccart-Gebhart et al. 2005). Trastuzumab has also shown a highly significant increase in disease-free survival and overall survival in three adjuvant trials (NCCTG N 9831, NSABP B-31 and HERA-trial) (Baselga et al. 2006; Viani et al. 2007). Comparisons suggest that trastuzumab given concurrently with taxanes may have superior efficacy than given sequentially after chemotherapy (Romond et al. 2005). In the neoadjuvant treatment, addition of trastuzumab to chemotherapy has increased pathological complete response (pCR) from 26\% (P (paclitaxel) $\times 4$ followed by FEC (fluorouracil/epirubicin/ cyclophosphamide $) \times 4)$ to $65 \% \quad(\mathrm{P} \times 4$ followed by FEC $\times 4$ together with trastuzumab) (Buzdar et al. 2005) with pCR still being a marker of good survival (Lazaridis et al. 2008). Thus, the fate of patients with HER2/neu-positive disease has been improved, but eventually most patients relapse (Colozza et al. 2007).

To date, it is not known how long trastuzumab should be given, whether cells become resistant to trastuzumab due to loss of receptor expression (Nahta and Esteva 2006) or to a modified receptor, no longer accessible to the antibody 
(Kumar 2007) or other ways of resistance (Berns et al. 2007; Pályi-Krekk et al. 2007).

This has prompted research for other molecules still active when trastuzumab does not work any more (Cardoso et al. 2002). Lapatinib (Mukherjee et al. 2007) is such a molecule, a dual tyrosine kinase inhibitor that blocks phosphorylation of both epidermal growth factor receptor (EGFR) and HER2/neu (Nahta et al. 2007). Also, lapatinib is specifically and exclusively bound to these receptors and has a slow off-rate for both ErbB1 and ErbB2 (Ito et al. 2007). Preclinical studies showed that lapatinib may cause apoptosis in HER2/neu-overexpressing cells even if the cells have been previously exposed to trastuzumab (Konecny et al. 2006). In clinical trials, objective responses were obtained in heavily pre-treated patients with HER2/neu-overexpressing metastatic breast cancer (Burris 2004) and a combination of lapatinib and capecitabine was superior to capecitabine alone (Tripathy 2007). We present here two cases of heavily pre-treated patients in whom trastuzumab was not capable of eliminating the CETC in the blood and the disease progressed. But treatment with a combination of capecitabine and lapatinib led to immediate elimination of all CETC.

\section{Methods}

Two patients were monitored for CETC and the response of these cells to the different therapeutic regimen during the course of disease. One millilitre blood samples anticoagulated with ethylene diamine tetra acidic acid (EDTA) were drawn before each new therapeutic manipulation and at each visit during subsequent observation.

Red blood cells were lysed with ammonium chloride (Qiagen, Hilden, Germany). For detection of CETC white cells from the sediment were subject to the MAINTRAC ${ }^{\circledR}$ analysis diluting the pellet in $500 \mu \mathrm{l}$ phosphate buffered salt (PBS) solution pH 7.4 adding $12.5 \mu \mathrm{l}$ of fluoresein-isothiocyanate (FITC)-conjugated mouse anti-human epithelial antibody (HEA) and $5 \mu$ phycoerythrin (PE) labelled antiCD45 (Miltenyi Bergisch Gladbach, Germany) simultaneously for $15 \mathrm{~min}$ in the dark. Analysis of red and green fluorescence of the cells was performed using a Laser Scanning Cytometer $^{\circledR}$ (Compucyte Corporation, Cambridge, MA, USA) enabling relocation of cells for visual examination of vital epithelial cells as extensively described previously (Pachmann et al. 2005b) or an image analysis system of Olympus ScanR which gave identical results.

A defined volume of the cell suspension was applied to a defined area on a slide or in a microtiter well and laser scanning or image analysis started of this area. Cells were detected by their forward scatter property, and red and green fluorescence recorded in the LSC or images taken in the ScanR. Epithelial cells stained with green fluorescence, exclusively surface-located, and normal blood cells stained with CD45 red fluorescence (Fig. 1). The epithelial cells selected by their green fluorescence were relocated and analysed for vitality. Only vital cells were counted.

Numbers of CETC were calculated per $\mathrm{ml}$ and varied between undetectable and 100,000. These numbers are tenfold higher than numbers obtained using the CellSearch system (Meng et al. 2004) but with more recent system numbers getting close to our results are obtained (Nagrath et al. 2007). The difference may be due to omission of magnetic separation which leads to a loss of specific cells together with the enrichment process, as discussed extensively in a previous study (Pachmann et al. 2005b). Normal blood cells could easily be distinguished from epithelial antigen-positive cells. No live epithelial cells were detected in $97 \%$ of healthy donors and in 38/40 patients with haematological malignancies (Pachmann et al. 2005b, Camara et al. 2007).

\section{Results}

The first patient treated with neoadjuvant chemotherapy was a poor responder according to our criteria published in Camara et al. (2007) with hardly a tenfold reduction in CETC and a subsequent re-increase. CETC numbers changed only marginally during the combined taxane/trastuzumab treatment typically associated with early relapse in HER2/neu-positive patients according to our observations. She then had surgery and subsequent treatment with trastuzumab for 1 year. Brain metastases detected already at diagnosis progressed after surgery and required radiotherapy. Still, trastuzumab was given 3-weekly as planned for 1 year. CETC numbers slowly started to decrease during the year of trastuzumab treatment (Fig. 2). Stable disease was observed during this year.

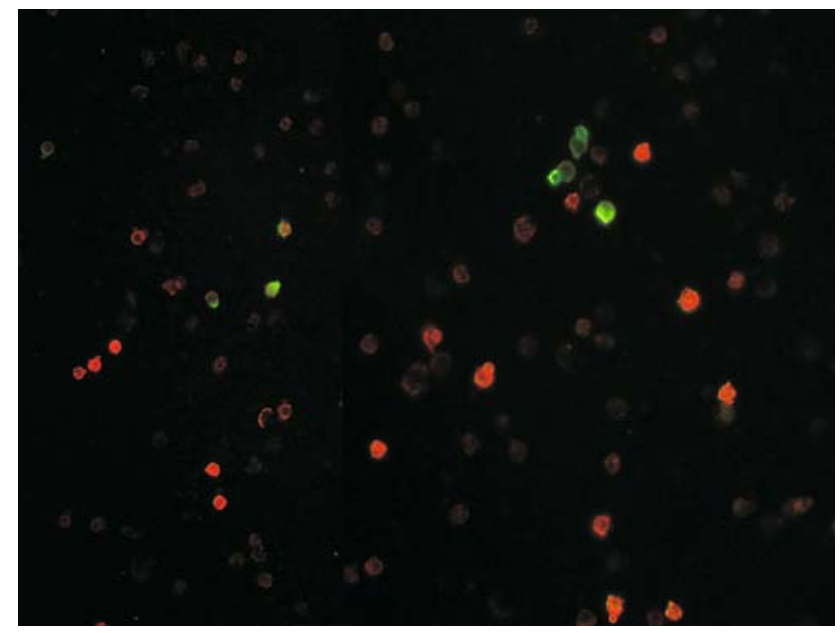

Fig. 1 Typical epithelial antigen positive cells with green caps among blood leucocytes stained orange for CD45 (250× magnification) 
Trastuzumab was halted as planned whereupon she developed skin metastases and her brain metastases progressed. She, therefore, received capecitabine for six courses together with lapatinib. Already upon the first cycle of capecitabine/lapatinib cell numbers decreased to below the threshold of detection and remained undetectable for the next 12 weeks but then started re-increasing together with a renewed progress of the brain metastases (Fig. 2).

In the second patient, after breast-conserving surgery, her CETC levels in blood rose to extremely high values. She was treated with adjuvant chemotherapy with EC and taxane/trastuzumab. Subsequently, she was treated for one more year of trastuzumab within the HERA-trial with an increase in CETC (Fig. 3). Trastuzumab was stopped after 1 year and a local relapse with skin involvement was diagnosed 2 months later which was treated with mastectomy (Fig. 3). Trastuzumab was re-initiated for 3 months but she again developed skin metastases during the following time which eventually required surgery. She then received capecitabine/lapatinib for six cycles upon which her CETC numbers fell to below the threshold of detection and have remained that low until now (Fig. 3). Her metastases have not shown further progress.

\section{Conclusions}

Monitoring of these CETC has shown to provide a valuable tool for analysis of the effect of therapeutic agents (Camara et al. 2007; Lobodasch et al. 2007). Thus, the response of CETC to the first cycles of neoadjuvant therapy highly correlates with tumour size reduction (Pachmann et al. 2005a) and this also results in an improved relapse-free survival in these patients (Camara et al. 2007), whereas lack of response in the neoadjuvant setting to anthracycline is a strong predictor of relapse independent of further treatment.

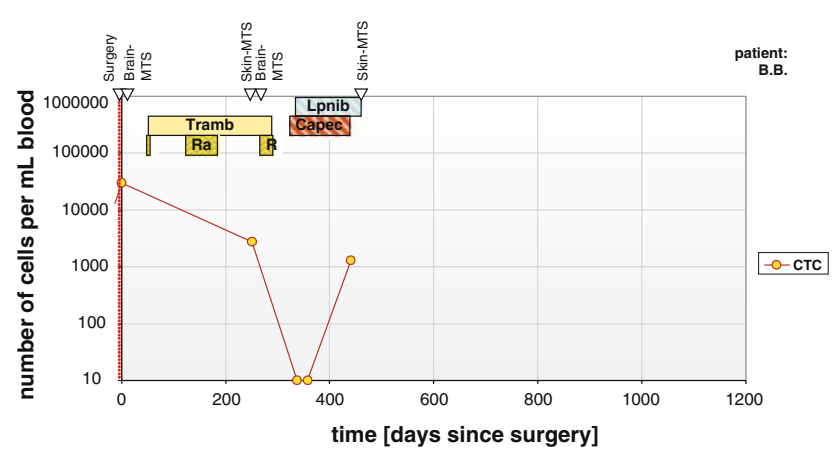

Fig. 2 Response of CETC from the first patient to therapy: minor decrease in CETC during subsequent trastuzumab therapy with concomitant development of further metastases and a rapid but short lasting decrease of CETC numbers during combined lapatinib and capecitabine therapy

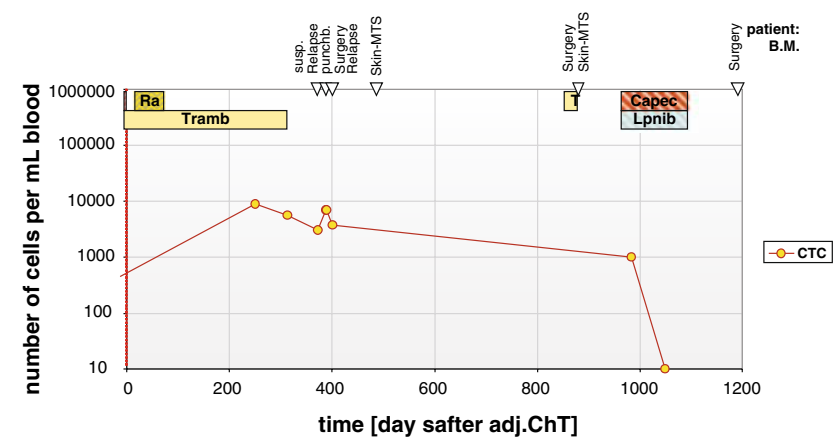

Fig. 3 Response of CETC from the second patient to therapy: increase in CETC during subsequent trastuzumab therapy with development of local relapse treated with mastectomy and a minor decrease during additional trastuzumab therapy with development of skin metastases. After surgery of the skin metastases rapid decrease of CETC numbers during combined lapatinib and capecitabine therapy occurs

This was, indeed, also true for the first patient, who presented with an inflammatory breast cancer, which is frequently accompanied by HER2/neu-positivity (GonzalezAngulo et al. 2007). She had very high CETC numbers from the beginning and already initially presented with brain metastases. FEC was only able to reduce her CETC moderately and the following combined treatment of taxane and trastuzumab led to a re-increase in CETC. We interpreted this as release of cells from the primary tumour during tumour tissue disintegration. Cells in the brain that had settled already early showed growth (Lin and Winer 2007) developing as progressive brain metastases in spite of subsequent trastuzumab treatment and more brain metastases appeared at the end of trastuzumab treatment. Initially, no extra-cranial growth was observed during the trastuzumab treatment (Kirsch et al. 2005), but CETC numbers only marginally decreased without being completely eliminated. CETC presented at diagnosis or released during neoadjuvant therapy may have contained a subpopulation of cells that were only temporarily silenced by trastuzumab treatment but eventually were able to metastasize and skin metastases developed. Due to disease progress capecitabine/lapatinib therapy (Bilancia et al. 2007) was initiated, and there was an immediate and complete elimination of CETC indicating an excellent response to this treatment (Pachmann et al. 2008) at least in the peripherally circulating cells. This did, however, not translate into a long-lasting reduction of metastatic disease and additional skin metastases appeared ( $<4$ months) accompanied by reappearance of CETC.

The second patient showed an extremely high CETC dissemination after surgery and relapsed early after adjuvant chemotherapy. Cell numbers remained high and even increased during the following trastuzumab treatment. Thus trastuzumab was not able to eliminate circulating tumour cells. But its ability to drive tumour cells into G1 (G0) of 
the cell cycle (Brockhoff et al. 2007) may have been responsible for prohibiting the cells from growing into detectable relapse. Metastatic cells may already have settled during the massive release of cells following surgery, so that a local relapse became apparent shortly after halting the adjuvant trastuzumab therapy. Again CETC responded immediately and consistently to the capecitabine/lapatinib combination and completely disappeared.

We have presented here two cases of patients with HER2/neu-positive breast cancer who showed progress during treatment with trastuzumab. In both cases, CETC showed no, or only marginal, reduction during treatment with the antibody, indicating that in these patients trastuzumab possibly inhibited cell growth but was not capable of eliminating CETC. Thus, binding of the antibody to the relevant cells seems not or hardly to induce apoptosis or cell death but instead may prevent cells in blood from settling and perhaps multiplying.

A final trial with capecitabine and lapatinib for the first time completely eliminated all CETC in the first patient with an initial diagnosis of inflammatory breast cancer.

Even if treatment was no longer effective in the manifest skin metastases, our results contribute indications that lapatinib is effective in combination with capecitabine (Geyer et al. 2006) and indeed leads to cell elimination (Spector et al. 2005) even in inflammatory breast cancer (Cristofanilli et al. 2006).

The same was true for the second patient who received trastuzumab after adjuvant treatment. Her circulating cells showed an increase in CETC numbers during trastuzumab with local relapse. Effective elimination was only observed with the combination of capecitabine and lapatinib, leading to cell elimination possibly by cell death due to effective inhibition of signalling pathways (Wood et al. 2004).

Our results proved the first observation that the antiHER2/neu antibody results in a temporary proliferation stop but rarely completely eliminates the tumour cells. These cells may be responsible for eventual metastasis formation after antibody withdrawal. In contrast, lapatinib may, especially in the context of additional cell toxic drugs, induce cell death under clinical relevant conditions.

Therefore, these results might warrant earlier application of lapatinib, for example, in combination with capecitabine in the clinical setting in order to eliminate the source of metastases, the CETC, before they can settle and then may become less accessible to elimination. Treatment success can be controlled monitoring the response of CETC.

Open Access This article is distributed under the terms of the Creative Commons Attribution Noncommercial License which permits any noncommercial use, distribution, and reproduction in any medium, provided the original author(s) and source are credited.

\section{References}

Baselga J, Perez EA, Pienkowski T et al (2006) Adjuvant trastuzumab: a milestone in the treatment of HER-2-positive early breast cancer. Oncologist 11:4-12. doi:10.1634/theoncologist.11-90001-4

Berns K, Horlings HM, Hennessy BT et al (2007) A functional genetic approach identifies the PI3 K pathway as a major determinant of trastuzumab resistance in breast cancer. Cancer Cell 12:395-402. doi:10.1016/j.ccr.2007.08.030

Bilancia D, Rosati G, Dinota A et al (2007) Lapatinib in breast cancer. Ann Oncol 18(Suppl 6):vi26-vi30. doi:10.1093/annonc/mdm220

Brockhoff G, Heckel B, Schmidt-Bruecken E et al (2007) Differential impact of cetuximab, pertuzumab and trastuzumab on BT474 and SK-BR-3 breast cancer cell proliferation. Cell Prolif 40:488-507. doi:10.1111/j.1365-2184.2007.00449.x

Burris HAIII (2004) Dual kinase inhibition in the treatment of breast cancer: initial experience with the EGFR/ErbB-2 inhibitor lapatinib. Oncologist 9(Suppl. 3):10-15. doi:10.1634/theoncologist.9suppl_3-10

Buzdar AU, Ibrahim NK, Francis D et al (2005) Significantly higher pathologic complete remission rate after neoadjuvant therapy with trastuzumab, paclitaxel, and epirubicin chemotherapy: results of a randomized trial in human epidermal growth factor receptor 2-positive operable breast cancer. J Clin Oncol 23:36763685. doi:10.1200/JCO.2005.07.032

Camara O, Rengsberger M, Egbe et al (2007) The relevance of circulating epithelial tumour cells (CETC) for therapy monitoring during neoadjuvant (primary systemic) chemotherapy in breast cancer. Ann Oncol 18:1484-1492. doi:10.1093/annonc/mdm206

Cardoso F, Piccart MJ, Durbecq V et al (2002) Resistance to trastuzumab: a necessary evil or a temporary challenge? Clin Breast Cancer 3:247-257

Cristofanilli M, Boussen H, Baselga J et al (2006) A phase II combination study of lapatinib and paclitaxel as a neoadjuvant therapy in patients with newly diagnosed inflammatory breast cancer (IBC). Breast Cancer Res Treat 100(Suppl. 1):S5\#1

Colozza M, de Azambuja E, Personeni N et al (2007) Achievements in systemic therapies in the pregenomic era in metastatic breast cancer. Oncologist 12:253-270. doi:10.1634/theoncologist. 12-3-253

Geyer CE, Forster J, Lindquist D et al (2006) Lapatinib plus capecitabine for Her2-positive advanced breast cancer. N Engl J Med 355:2733-2743. doi:10.1056/NEJMoa064320

Gonzalez-Angulo AM, Hennessy BT, Broglio K et al (2007) Trends for inflammatory breast cancer: is survival improving? Oncologist 12:904-912. doi:10.1634/theoncologist.12-8-904

Ito Y, Tokudome N, Sugihara T et al (2007) Does lapatinib, a smallmolecule tyrosine kinase inhibitor, constitute a breakthrough in the treatment of breast cancer? Breast Cancer 14:156-162. doi: $10.2325 /$ jbcs. 971

Kirsch DG, Ledezma CJ, Mathews CS et al (2005) Survival after brain metastases from breast cancer in the trastuzumab era. J Clin Oncol 23:2114-2116. doi:10.1200/JCO.2005.05.249

Konecny GE, Pegram MD, Venkatesan N et al (2006) Activity of the dual kinase inhibitor lapatinib (GW572016) against HER-2-overexpressing and trastuzumab-treated breast cancer cells. Cancer Res 66:1630-1639. doi:10.1158/0008-5472.CAN-05-1182

Kumar R (2007) ErbB-dependent signaling as a determinant of trastuzumab resistance. Clin Cancer Res 13:4657-4659. doi:10.1158/ 1078-0432.CCR-07-1401

Lazaridis G, Pentheroudakis G, Pavlidis N (2008) Integrating trastuzumab in the neoadjuvant treatment of primary breast cancer: accumulating evidence of efficacy, synergy and safety. Crit Rev Oncol Hematol 66:31-41. doi:10.1016/j.critrevonc.2007. 07.002 
Lin NU, Winer EP (2007) Brain metastases: the HER2 paradigm. Clin Cancer Res 13:1648-1655. doi:10.1158/1078-0432.CCR-062478

Lobodasch K, Fröhlich F, Rengsberger M et al (2007) Quantification of circulating tumour cells for monitoring of adjuvant therapy in breast cancer: an increase in cell number at completion of therapy is a predictor of early relapse. Breast 16:211-218. doi:10.1016/ j.breast.2006.12.005

Meng S, Tripathy D, Frenkel EP et al (2004) Circulating tumor cells in patients with breast cancer dormancy. Clin Cancer Res 10:81528162. doi:10.1158/1078-0432.CCR-04-1110

Mukherjee A, Dhadda AS, Shehata M et al (2007) Lapatinib: a tyrosine kinase inhibitor with a clinical role in breast cancer. Expert Opin Pharmacother 8:2189-2204. doi:10.1517/14656566.8.13.2189

Nagrath S, Sequist LV, Maheswaran S et al (2007) Isolation of rare circulating tumour cells in cancer patients by microchip technology. Nature 450:1235-1239. doi:10.1038/nature06385

Nahta R, Esteva FJ (2006) Herceptin: mechanisms of action and resistance. Cancer Lett 232:123-138. doi:10.1016/j.canlet.2005. 01.041

Nahta R, Yuan LXH, Du Y et al (2007) Lapatinib induces apoptosis in trastuzumab-resistant breast cancer cells: effects on insulin-like growth factor I signalling. Mol Cancer Ther 6:667-674. doi:10.1158/1535-7163.MCT-06-0423

Pachmann K, Camara O, Kavallaris A et al (2005a) Quantification of the response of circulating epithelial cells (CEC) to neodadjuvant treatment of breast cancer: a new tool for therapy monitoring. Breast Cancer Res 7:R975-R979. doi:10.1186/bcr1328

Pachmann K, Clement JH, Schneider CP et al (2005b) Standardized quantification of circulating peripheral tumor cells from lung and breast cancer. Clin Chem Lab Med 43:617-627. doi:10.1515/ CCLM.2005.107

Pachmann K, Camara O, Kavallaris A et al (2008) Monitoring the response of circulating epithelial tumor cells (CETC) to adjuvant chemotherapy in breast cancer allows detection of patients at risk of early relapse. J Clin Oncol 26:1208-1215. doi:10.1200/ JCO.2007.13.6523

Pályi-Krekk Z, Barok M, Isola J et al (2007) Hyaluronan-induced masking of ErbB2 and CD44-enhanced trastuzumab internalisation in trastuzumab resistant breast cancer. Eur J Cancer 43:2423 2433. doi:10.1016/j.ejca.2007.08.018

Piccart-Gebhart MJ, Procter M, Leyland-Jones B et al (2005) Herceptin Adjuvant (HERA) Trial Study Team. Trastuzumab after adjuvant chemotherapy in HER2-positive breast cancer. N Engl J Med 353:1659-1672. doi:10.1056/NEJMoa052306

Romond EH, Perez EA, Bryant J et al (2005) Trastuzumab plus adjuvant chemotherapy for operable HER2-positive breast cancer. N Engl J Med 353:1673-1684. doi:10.1056/NEJMoa052122

Seidman AD, Fornier MN, Esteva FJ et al (2001) Weekly trastuzumab and paclitaxel therapy for metastatic breast cancer with analysis of efficacy by HER 2 immunophenotype and gene amplification. $\mathrm{J}$ Clin Oncol 19:2587-2595

Spector NL, Xia W, Burris H 3rd (2005) Study of the biologic effects of lapatinib, a reversible inhibitor of ErbB1 and ErbB2 tyrosine kinases, on tumor growth and survival pathways in patients with advanced malignancies. J Clin Oncol 23:2502-2512. doi:10.1200/JCO.2005.12.157

Tripathy D (2007) Capecitabine in combination with novel targeted agents in the management of metastatic breast cancer: underlying rationale and results of clinical trials. Oncologist 12:375-389. doi:10.1634/theoncologist.12-4-375

Viani GA, Afonso SL, Stefano EJ et al (2007) Adjuvant trastuzumab in the treatment of her-2-positive early breast cancer: a meta-analysis of published randomized trials. BMC Cancer 7:153. doi:10.1186/1471-2407-7-153

Wood ER, Truesdale AT, McDonald OB et al (2004) A unique structure for epidermal growth receptor bound to GW572016 (lapatinib): relationships among protein conformation, inhibitor off-rate, and receptor activity in tumor cells. Cancer Res 64:6652-6659. doi:10.1158/0008-5472.CAN-04-1168 